\title{
RELACIONES BIOLÓGICAS EN GRUPOS CAZADORES- RECOLECTORES DE LA TRANSICIÓN PAMPEANO-PATAGÓNICA ORIENTAL (ARGENTINA) DURANTE EL HOLOCENO TARDÍO. APORTES DESDE LA MÉTRICA DENTAL
}

\author{
Leandro H. Luna1*; Gustavo Flensborg² y Gustavo Martínez²
}

\begin{abstract}
${ }^{I}$ IDECU-CONICET. Museo Etnográfico J. B. Ambrosetti. Facultad de Filosofía y Letras. Universidad de Buenos Aires. Cuidad Autónoma de Buenos Aires. Argentina

${ }^{2}$ INCUAPA-CONICET-UNICEN. Facultad de Ciencias Sociales. Universidad Nacional del Centro de la Provincia de Buenos Aires. Olavarría. Argentina
\end{abstract}

PALABRAS CLAVE Pampa; Norpatagonia; antropología dental; dinámicas poblacionales; continuidad fenotípica

\begin{abstract}
RESUMEN Los modelos bioantropológicos y arqueológicos proponen cambios en la demografía, en la organización social, en las dinámicas poblacionales y en las redes de interacción social entre los grupos cazadores-recolectores del norte de la Patagonia y del sur pampeano durante los últimos 1000 años. El objetivo de este trabajo es explorar, desde la perspectiva de la antropología dental, si los individuos recuperados en el curso inferior del río Colorado (transición pampeano-patagónica oriental, provincia de Buenos Aires), inhumados durante el Holoceno tardío (ca. 3000-250 años AP), pertenecieron a diferentes poblaciones biológicas o si, por el contrario, compartieron un pool génico común a lo largo de ese período. Para alcanzar este objetivo se realizaron análisis métricos de la dentición permanente de 21 individuos
\end{abstract}

adultos de ambos sexos, procedentes de tres sitios arqueológicos. Los resultados indican una similitud en la dispersión de los valores obtenidos, lo que permitiría inferir cierta continuidad fenotípica. En este sentido, no se observan segregaciones claras entre grupos de individuos, de manera que es posible plantear que no habrían existido divergencias biológicas entre los grupos humanos que inhumaban a sus ancestros en el área durante todo el lapso considerado. Estos datos contrastan con la información generada en áreas vecinas (p. ej., Pampa occidental), lo que indicaría que durante el Holoceno tardío final (ca. 1000-250 años AP) se habrían desarrollado diferentes procesos y dinámicas poblacionales (p.ej., regionalización) a nivel macro-regional. Rev Arg Antrop Biol 19(2), 2017. doi:10.17139/raab.2017.0019.02.02

KEY WORDS Pampas; Northern Patagonia; dental anthropology; population dynamics; phenotypic continuity

ABSTRACT Bioanthropological and archaeological models propose changes in demography, social organization, population dynamics, and social network interactions among hunter-gatherer groups of northern Patagonia and southern Pampas over the last 1000 years BP. Following this argument, the aim of this paper is to examine, from a dental anthropological perspective, whether the individuals recovered in the lower course of the Colorado river (eastern Pampean-Patagonian transition, Buenos Aires province, Argentina), buried during the late Holocene (ca. 3000-250 years BP), belonged to different biological populations or, on the contrary, whether they shared a common gene pool throughout this period. To achieve this goal, dental metrics were performed on adults from both sexes, recovered from three archaeological sites. The results indicate a similarity in the dispersion of the values obtained, which would allow the inference of certain phenotypic continuity. In this sense, no clear segregations among groups of individuals are observed, so that it is possible to argue that biological differences would not have existed among the human groups that buried their ancestors in the lower course of the Colorado river during the period considered. These data contrast with the information generated in neighboring areas (e.g., western Pampas), indicating that during the final late Holocene (ca. 1000-250 years BP) different processes and population dynamics (e.g., regionalization) developed at a macro-regional level. Rev Arg Antrop Biol 19(2), 2017. doi: 10.17139/raab.2017.0019.02.02
La importancia de la antropología dental y los análisis métricos en bioarqueología y antropología forense se ha incrementado notablemente en las últimas décadas (Mayhall, 1992, 2000; Hillson, 1996; Pietrusewsky, 2000; Latham y Finnegan, 2010; Black y Ferguson, 2011; Luna, 2016), en especial en el campo vinculado a estudios de biodistancia (Buikstra et al., 1990; Lukacs, 1998; Kieser, 2008). Esto se debe a que la dentición ofrece un gran potencial para evaluar procesos migratorios y evolutivos, inte- racciones biológicas y dinámicas poblacionales (Scott y Turner, 1988, 2000; Pinhasi et al., 2008;

\footnotetext{
*Correspondencia a: Leandro H. Luna. CONICET. Museo Etnográfico J. B. Ambrosetti. Facultad de Filosofía y Letras. Universidad de Buenos Aires. Moreno 350. 1091 Ciudad Autónoma de Buenos Aires. Argentina. E-mail: lunaranda@ gmail.com
}

Financiamiento: PIP-CONICET 0122; PICT 242/2012.

Recibido 15 Junio 2016; aceptado 24 Octubre 2016

doi:10.17139/raab.2017.0019.02.02 
Matsumura y Oxenham, 2014; Bollini et al., 2016). Los análisis se fundamentan en que tanto el tamaño como la morfología dental se encuentran fuertemente influenciados por el genotipo, con una baja incidencia de factores externos (e.g., aspectos medioambientales o condicionamientos de índole social) en el desarrollo de los tejidos dentales (Cucina et al., 1999; Dempsey y Townsend, 2001; Harris et al., 2001; Hanihara e Ishida, 2005; Harris y Lease, 2005; Matsumura y Hudson, 2005). Como los dientes son los elementos del sistema esqueletal-dentario que más tempranamente adoptan su forma definitiva, el período de tiempo de su formación, en el que pueden verse afectados por factores ambientales es relativamente corto respecto de otros elementos que componen el sistema esqueletal. Sin embargo, la estructura dental puede verse afectada por factores externos como el desgaste dental o situaciones de salud bucal deficiente. A pasar de esto, los dientes constituyen un tipo de tejido esqueletal valioso para el estudio de las relaciones biológicas. Asimismo, dado su pequeño tamaño y su estructura tenaz, son los elementos del cuerpo humano más resistentes al deterioro postdepositacional, motivo por el cual suelen recuperarse en altas frecuencias, preservándose generalmente mejor que los restos óseos (Hillson, 1996; Luna, 2008, 2015).

En Argentina existen escasos antecedentes en el estudio métrico de los dientes humanos. Solo en los últimos años se han comenzado a desarrollar análisis uni y multivariados de biodistancia considerando variables métricas (Bernal, 2007, 2008; Luna, 2008, 2015; Bernal et al., 2010; Bollini et al., 2012). Las investigaciones se focalizaron principalmente en el relevamiento de muestras de grupos cazadores-recolectores que ocuparon las regiones de Pampa y Patagonia durante el Holoceno tardío. Entre los tópicos explorados se destacan los relacionados con el estudio de los patrones de variación biológica de las poblaciones humanas del Holoceno tardío final de Patagonia, con el propósito de discutir procesos y eventos que modelaron su historia evolutiva a partir del análisis de las medidas del cuello dental (Bernal, 2008). Asimismo, se evaluó la correspondencia entre los factores responsables de las variaciones métricas y variables de índole geográfica, molecular y ecológica (Bernal et al., 2010). También se realizaron aná- lisis sobre el tamaño y la forma de los molares, comparando la información obtenida a partir de las medidas lineales con la generada por procedimientos derivados de la morfometría geométrica (Bernal, 2007). En el sitio Chenque I (sur de la provincia de La Pampa; subregión Pampa Occidental), se evaluaron las variaciones en los patrones dimórficos de las medidas de la corona y del cuello dental para obtener información sobre el sexo y el número mínimo de individuos inhumados a partir de una muestra de restos humanos sin asociación anatómica (Luna, 2008). Con ese conjunto se realizaron también estudios métricos con el objetivo de mejorar las estimaciones de edad de muerte, efectuar un aporte para la caracterización paleodemográfica del sitio (Luna, 2006, 2008, 2010a, b, 2012) y evaluar el grado de incidencia de factores exógenos en el fenotipo dental (Luna, 2015). Asimismo, se realizó una primera aproximación al análisis de las distancias biológicas a nivel individual (Luna, 2008), lo que contribuyó a robustecer modelos previos de dinámica poblacional propuestos por Berón $(2004,2007 a, 2010)$ para dicha zona. Por último, Bollini et al. (2012) llevaron a cabo un estudio odontométrico comparativo de restos procedentes del Delta del Paraná (provincias de Buenos Aires y Entre Ríos), de la desembocadura del río Negro (provincia de Río Negro), y de los sitios Pampa Grande (provincia de Salta) y Arroyo Seco 2 (provincia de Buenos Aires), con el propósito de evaluar variaciones de las poblaciones humanas en el tiempo y espacio. Estos antecedentes muestran que los análisis métricos de la dentición constituyen una línea de trabajo que se encuentra en sus inicios en el país, y que contribuye al conocimiento de las interacciones sociales y de la intensidad de las relaciones biológicas intergrupales de poblaciones nativas americanas. Todos estos trabajos utilizaron las medidas dentales de la corona y del cuello aplicadas en el presente caso de estudio (ver Material y Métodos), aunque otorgando especial relevancia a las últimas debido al deterioro que suele sufrir la dentición de las poblaciones cazadoras-recolectoras (ver debajo). Asimismo, la literatura internacional abunda en ejemplos de investigaciones desarrolladas a partir del análisis de la métrica dental en poblaciones actuales y extintas, con el objetivo de responder preguntas similares, orientadas a identificar patrones 
de variación en las relaciones biológicas en diferentes escalas espacio-temporales (e.g. Harris y Nweeia, 1980; Christensen, 1998; Hanihara e Ishida, 2005; Harris y Lease, 2005; Matsumura y Hudson, 2005). Este trabajo es original en el sentido de que considera como unidad de análisis a cada individuo en particular y no a diferentes agrupaciones de individuos (en el sentido general propuesto por Alt y Vach, 1995).

En la transición pampeano-patagónica oriental, específicamente el curso inferior del río Colorado (Fig. 1), los entierros humanos son uno de los componentes más destacados del registro arqueológico asignable al Holoceno tardío (ca. 3000-250 años AP). A partir del estudio de las modalidades de entierro, estimaciones paleodemográficas e indicadores de salud (e.g., caries, osteoartrosis), en conjunto con otras líneas de análisis que incluyen la evaluación de la subsistencia, la organización de la tecnología lítica y cerámica, y los patrones de movilidad y asenta- miento, se propuso que hacia los ca. 1000 años AP se produjeron cambios importantes a nivel organizacional en los grupos cazadores-recolectores que habitaron la zona (Martínez, 20082009; Martínez et al., 2013, 2017; Stoessel y Martínez, 2014; Flensborg, 2015; Flensborg et al., 2015; Armentano, 2016). Estos cambios habrían estado vinculados a una serie de procesos sociales y biológicos, entre los que se destacan el aumento de la densidad demográfica en el noreste de Patagonia, una intensa dinámica poblacional a nivel macro-regional, constreñimiento espacial, competencia y comportamientos territoriales complejos, procesos de intensificación en la subsistencia y el desarrollo de redes de interacción social relativamente cerradas en el marco de un proceso de regionalización creciente. Aunque la frecuencia e intensidad de los contactos entre áreas de las regiones Pampeana, Patagónica y Cuyo se modificaron como consecuencia de este proceso de regionalización

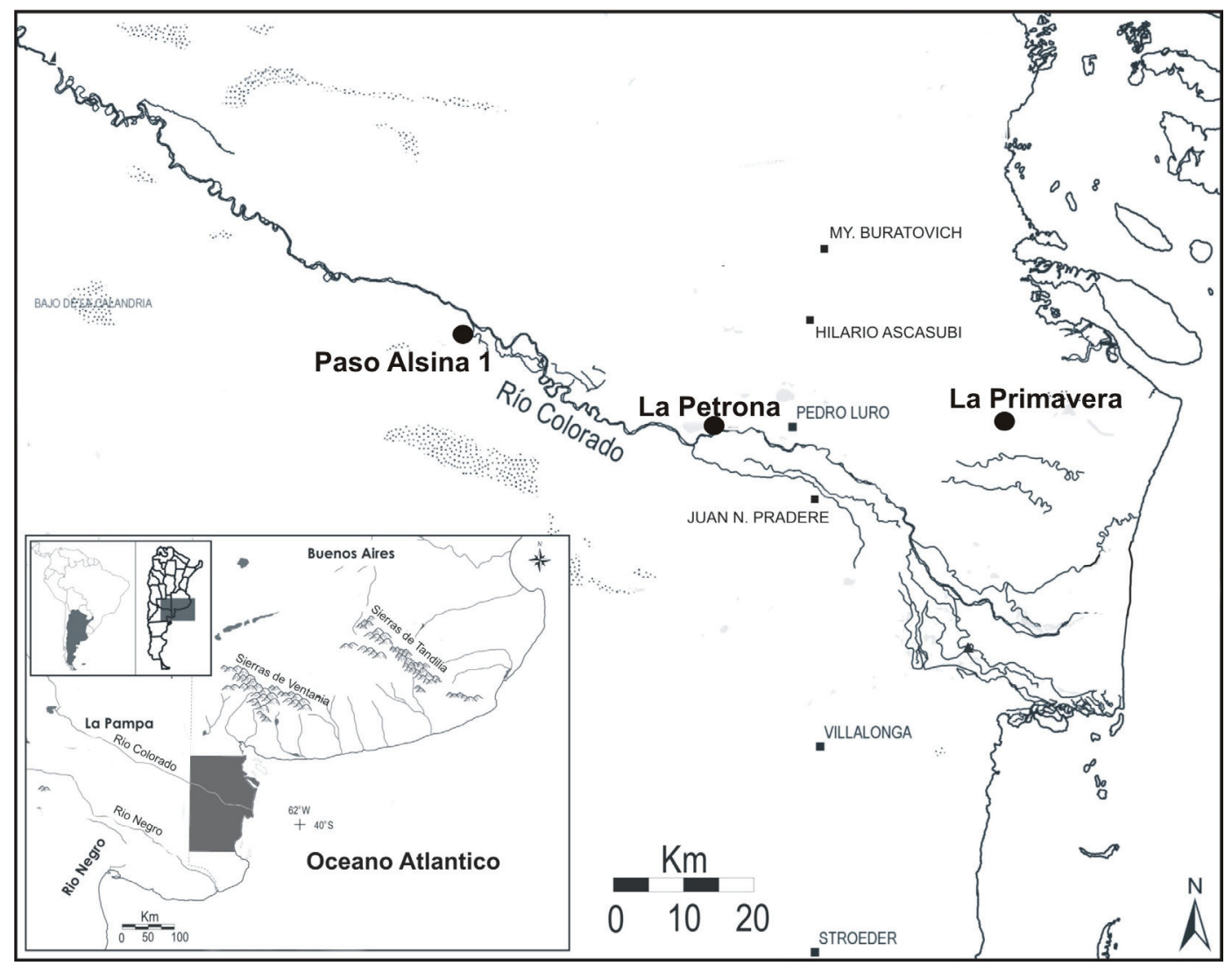

Fig. 1. Localización geográfica de los sitios arqueológicos analizados en el curso inferior del río Colorado. 
(Martínez et al., 2017), el sistema siempre se caracterizó por la presencia de relaciones de coexistencia y complementariedad social entre grupos (Berón, 2007b; Martínez, 2008-2009). En el marco de los cambios mencionados para los últimos $c a$. 1000 años AP, existen otras propuestas que, además de considerar estos últimos procesos (Berón, 2004), argumentan la existencia de reemplazos poblacionales y/o contracción demográfica de grupos (Barrientos, 2001; Barrientos y Perez, 2004, 2005).

El objetivo de este trabajo es presentar y discutir los resultados y las tendencias obtenidas a partir del análisis métrico dental de individuos recuperados en el curso inferior del río Colorado durante el Holoceno tardío, a los efectos de explorar si los mismos habrían pertenecido a diferentes poblaciones biológicas o si por el contrario compartieron un pool génico común en el transcurso de ese período. Asimismo, desde esta línea de análisis novedosa para el área de estudio y escasamente empleada en los estudios bioantropológicos de Argentina, se propone evaluar algunas características de los modelos de dinámica poblacional mencionados anteriormente.

\section{MATERIAL Y MÉTODOS}

Si bien en el área de estudio se han recuperado hasta el momento 94 individuos de diferentes edades de muerte y cronologías (Flensborg, 2015; Flensborg et al., 2015), los adultos son los que presentan, en la mayoría de los casos, la dentición más completa. De ese total, 26 fueron analizados en este trabajo, ya que son los que contienen al menos el $50 \%$ de los dientes en sus alveolos. A su vez, considerando las características de la muestra que se detallan más abajo, se seleccionaron 21 individuos: 9 femeninos, 9 masculinos y 3 indeterminados para el tratamiento de los datos métricos (Tabla 1). La serie dental proviene de los sitios arqueológicos La Primavera (LPR; $n=2)$, La Petrona (LPE; $n=3)$ y Paso Alsina 1 (PA; n=16) (Fig. 1). En La Primavera se recuperaron seis individuos asignables a una cronología de ca. 2900-2700 años AP. El sitio se corresponde funcionalmente con una base residencial de actividades múltiples, en la cual se llevaron a cabo inhumaciones (Bayón et al., 2004; Martínez, 2008-2009; Flensborg, 2012). En el sitio La Petrona se recuperaron ocho in- dividuos y la cronología obtenida ubica al contexto arqueológico en $c a$. 800-250 años AP. Martínez y Figuerero Torres (2000) lo definen como un campamento de actividades múltiples, recurrentemente ocupado para diversas funciones domésticas y funerarias (véase Flensborg et al., 2011). Por último, Paso Alsina 1 es un área formal de entierro de pequeñas dimensiones $\left(6 \mathrm{~m}^{2}\right)$, compuesta por 10 entierros secundarios múltiples de tipo fardo funerario y representada por 77 individuos de diferentes categorías etarias y ambos sexos (Flensborg et al., 2015). Los fechados radiocarbónicos obtenidos de diferentes entierros arrojaron edades similares entre sí, por lo que se estimó una media ponderada en $c a$. 500 años AP (Martínez et al., 2007). Para estos últimos dos sitios, diferentes análisis bioarqueológicos dan cuenta de una clara manipulación intencional en el procesamiento de cadáveres, selección de partes esqueletarias, tinción sobre las unidades óseas, armado de los paquetes funerarios, traslado y posterior entierro de individuos cuya procedencia muy probablemente exceda los límites del área de estudio, involucrando una escala espacial macro regional pampeano-norpatagónico (Martínez et al., 2006, 2012; González, 2013).

Para el análisis métrico se consideraron en primera instancia las medidas bucolinguales y mesiodistales de la corona y del cuello de los dientes permanentes que se comportan de manera más estable y presentan los menores coeficientes de variación de acuerdo con la literatura odontométrica; por lo tanto, son los que se verían menos afectados por el impacto de los estresores socio-ambientales (Butler, 1937, 1939; Dahlberg, 1945; Garn et al., 1967; Kieser, 2008; ver Luna, 2015). Se seleccionaron para este procedimiento las medidas de los segundos incisivos, los caninos, los primeros premolares y los primeros molares. El diámetro mesiodistal de la corona se midió considerando los puntos extremos mesial y distal de cada pieza dental, paralelo a la superficie oclusal, mientras que el diámetro bucolingual de la corona corresponde a la mayor distancia entre las superficies bucal y lingual, perpendicular a la medida anterior. Las medidas del cuello se midieron para cada diente a la altura de la unión del esmalte con el cemento, paralelas a las medidas máximas de la corona. El diámetro bucolingual del cuello es la 
TABLA 1. Medidas del cuello dental (en milímetros)

\begin{tabular}{|c|c|c|c|c|c|c|c|c|c|c|c|}
\hline \multirow[b]{2}{*}{ Sitio } & \multirow[b]{2}{*}{ Individuo } & \multirow[b]{2}{*}{ Sexo } & \multirow[b]{2}{*}{$\begin{array}{c}\text { Edad } \\
\text { (años) }\end{array}$} & \multicolumn{2}{|c|}{ 2IS } & \multicolumn{2}{|c|}{$\mathrm{CS}$} & \multicolumn{2}{|c|}{$1 \mathrm{PmS}$} & \multicolumn{2}{|c|}{$1 \mathrm{MS}$} \\
\hline & & & & MD & $\mathrm{BL}$ & MD & $\mathrm{BL}$ & MD & $\mathrm{BL}$ & $\mathrm{MD}$ & $\mathrm{BL}$ \\
\hline \multirow[b]{2}{*}{ La Primavera } & LPR1 & $\mathrm{F}$ & $40-45$ & 4,69 & 5,47 & 5,55 & 6,99 & 4,54 & 7,43 & 8,05 & 10,72 \\
\hline & LPR2 & M & $20-25$ & 3,68 & 5,26 & 6,22 & 7,80 & 4,93 & 7,91 & 4,99 & 7,27 \\
\hline \multirow{3}{*}{ La Petrona } & LPE1 & $\mathrm{F}$ & $35-45$ & 4,79 & 5,45 & 6,13 & 7,72 & 4,74 & 7,90 & 7,83 & 10,41 \\
\hline & LPE2 & $\mathrm{F}$ & $35-45$ & 5,29 & 6,57 & 6,19 & 7,59 & 5,49 & 8,66 & 8,17 & 10,17 \\
\hline & LPE3 & F & $25-35$ & 5,44 & 6,24 & 6,33 & 8,13 & 5,12 & 9,20 & 7,97 & 12,29 \\
\hline \multirow{16}{*}{ Paso Alsina 1} & PA2-1 & $\mathrm{F}$ & $35-45$ & 5,28 & 6,01 & 5,52 & 7,65 & 5,07 & 7,17 & 7,83 & 10,40 \\
\hline & PA2-3 & M & $30-40$ & 5,35 & 5,28 & 6,27 & 8,07 & 5,44 & 8,10 & 8,78 & 11,07 \\
\hline & PA2-32 & M & $20-30$ & 5,41 & 5,63 & 6,49 & 8,08 & 5,06 & 7,70 & 8,81 & 11,59 \\
\hline & PA3-39 & M & $25-35$ & 5,35 & 6,50 & 6,49 & 8,08 & 5,17 & 8,74 & 7,59 & 11,07 \\
\hline & PA3-41 & $\mathrm{F}$ & $25-35$ & 5,51 & 5,80 & 5,96 & 7,86 & 5,00 & 7,91 & 8,07 & 10,40 \\
\hline & PA4-6 & M & $20-30$ & 5,20 & 6,15 & 5,78 & 7,92 & 5,11 & 8,54 & 8,87 & 11,18 \\
\hline & PA4-47 & M & $20-30$ & 5,20 & 5,90 & 7,52 & 8,12 & 7,62 & 9,44 & 9,42 & 12,48 \\
\hline & PA5-51 & M & $25-35$ & 5,20 & 5,90 & 6,49 & 8,08 & 5,58 & 7,40 & 8,80 & 10,62 \\
\hline & PA6-55 & $\mathrm{F}$ & $30-40$ & 5,26 & 5,97 & 5,49 & 7,51 & 5,33 & 8,07 & 9,37 & 10,30 \\
\hline & PA10A-9 & M & $18-25$ & 5,54 & 6,60 & 6,82 & 8,57 & 5,95 & 10,05 & 8,59 & 11,71 \\
\hline & PA10A-21 & $\mathrm{F}$ & $30-40$ & 5,83 & 6,26 & 6,20 & 9,04 & 4,72 & 7,95 & 8,01 & 10,40 \\
\hline & PA10A-24 & IN & $20-30$ & 5,20 & 5,96 & 6,19 & 7,94 & 8,99 & 11,57 & 5,06 & 9,10 \\
\hline & PA10A-26 & IN & $25-35$ & 5,26 & 5,96 & 6,69 & 8,72 & 5,50 & 7,64 & 9,13 & 12,10 \\
\hline & PA10A-27 & IN & $20-30$ & 5,20 & 5,97 & 6,81 & 7,52 & 5,21 & 9,16 & 9,02 & 11,94 \\
\hline & PA10A-34 & $\mathrm{F}$ & $20-30$ & 5,75 & 6,35 & 6,32 & 8,25 & 5,69 & 8,79 & 5,23 & 8,55 \\
\hline & PA10A-35 & M & $20-30$ & 5,11 & 5,90 & 6,38 & 8,49 & 5,36 & 7,82 & 8,53 & 11,98 \\
\hline
\end{tabular}

Referencias: 2IS:segundo incisivo superior; CS:canino superior; 1PmS:primer premolar superior; 1MS:primer molar superior; MD:diámetro mesiodistal; BL:diámetro bucolingual. Para los casos correspondientes a Paso Alsina 1, los números ubicados antes del guión, corresponden al entierro y los posteriores, al individuo.

mayor distancia existente entre los sectores bucal y lingual, y el mesiodistal, la distancia entre dos líneas paralelas imaginarias, perpendiculares al eje mesiodistal y tangenciales a los puntos extremos mediales y distales más oclusales de la unión del esmalte con el cemento (Mayhall, 1992, 2000; Hillson et al., 2005; Kieser, 2008) (Fig. 2).

Los dientes fueron medidos por uno de los autores (LHL) para evitar errores interobservador, utilizando un calibre digital Mitutoyo, Modelo 573-721, especialmente diseñado para análisis odontométrico. El error intraobservador fue previamente analizado eligiendo un total de
40 dientes completos, los cuales fueron medidos en dos oportunidades separadas por dos semanas. Los resultados obtenidos fueron analizados mediante el Coeficiente de Correlación Intraclase con el programa R 1.9.1. En ningún caso se observaron diferencias apreciables entre las dos mediciones obtenidas para cada variable, ya que los valores obtenidos fueron superiores a 0,90 (Luna, 2008).

Se constató que los porcentajes de datos perdidos para las medidas coronales superaban ampliamente los referidos a los diámetros del cuello dental, lo cual es un derivado directo del alto grado de desgaste presente en la mayoría de 


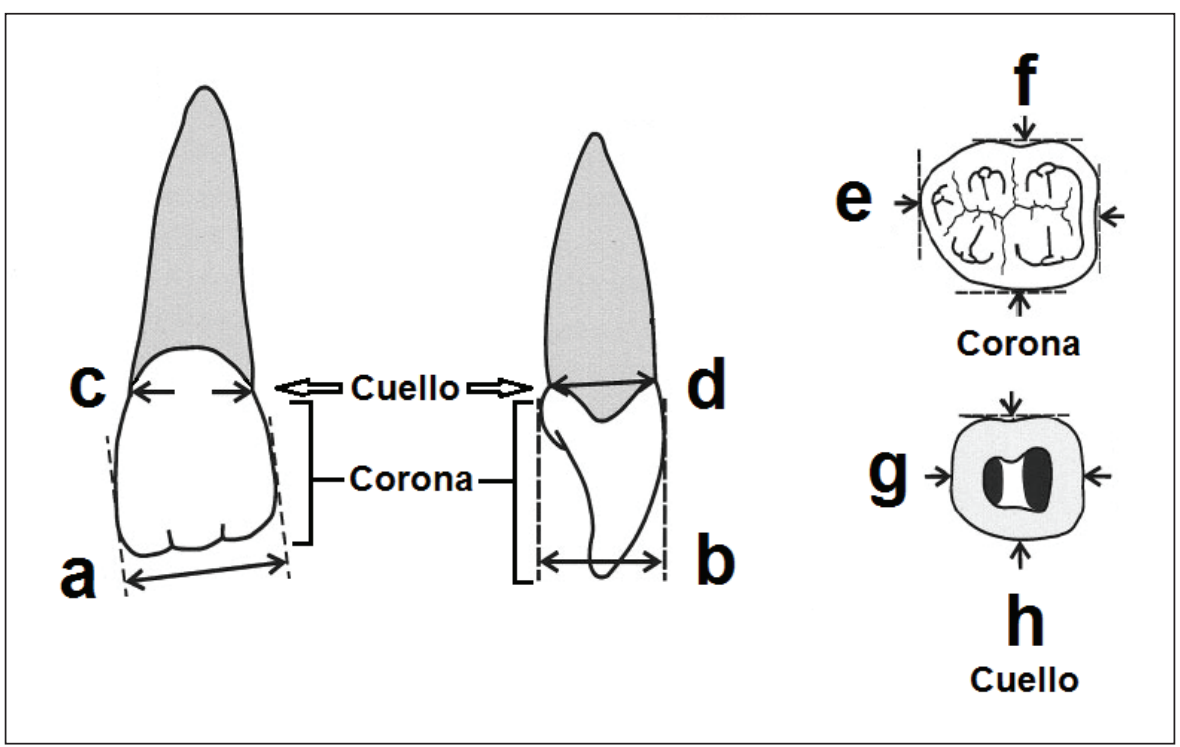

Fig. 2. Medidas dentales consideradas en este trabajo. a: diámetro mesiodistal de la corona de dientes unirradiculares; b: diámetro bucolingual de la corona de dientes unirradiculares; c: diámetro mesiodistal del cuello de dientes unirradiculares; d: diámetro bucolingual del cuello de dientes unirradiculares; e: diámetro mesiodistal de la corona de molares; f: diámetro bucolingual de la corona de molares; g: diámetro mesiodistal del cuello de molares; h: diámetro bucolingual del cuello de molares. Modificado de Hillson et al. (2005:Figs. 1 y 2).

las piezas dentales. Esta disparidad es recurrentemente identificada en estudios realizados con muestras de cazadores-recolectores, lo que restringe el uso de las medidas de la corona, especialmente de los diámetros mesiodistales (Luna, 2015). De esta manera, finalmente en este estudio, sólo se consideraron las medidas del cuello de los dientes antes mencionados. Por último, también se constató una menor representación de piezas dentales inferiores, consecuencia de que en los entierros analizados fueron recuperadas pocas mandíbulas. Por este motivo, teniendo en cuenta la alta cantidad de datos perdidos para dichos elementos, fueron excluidos del estudio y solamente se tuvieron en cuenta los dientes superiores. En total, de los 21 individuos se incluyeron 77 dientes en el análisis (18 segundos incisivos, 19 caninos, 20 primeros premolares y 20 primeros molares). Los datos perdidos $(n=7)$ fueron inferidos a través del procedimiento de imputación múltiple (Rubin, 1987) utilizando el programa NORM 2.03 (Schafer, 2000) y posteriormente incluidos en los análisis.

Los datos obtenidos fueron corregidos mediante la aplicación de las variables de forma de Mosimann (Jungers et al., 1995), procedimiento que permite una corrección de tamaño isométri- co. Cada medida lineal $\alpha$ es dividida por la media geométrica mg, y es calculada considerando todos los dientes de cada individuo; esta es una medida general de tamaño geométrico $(b=\alpha /$ $\mathrm{mg}$ ). Los resultados obtenidos maximizan la información sobre la morfología, mientras que se descarta gran parte de la influencia del tamaño en los resultados (Darroch y Mosimann, 1985; Jungers et al., 1995). De esta manera, los patrones dimórficos son minimizados y ambos sexos pueden ser comparados en un mismo conjunto.

La información obtenida para las ocho variables consideradas (diámetros mesiodistales y bucolinguales del cuello de segundos incisivos, caninos, primeros premolares y primeros molares superiores) fue reducida a través de un análisis de componentes principales (ACP) (Hair et al., 1999). Los primeros tres componentes principales (CP) fueron representados mediante dos gráficos bivariados. Los resultados también fueron analizados mediante un dendrograma jerárquico (método de conglomeración de vinculación intergrupos, utilizando la medida de distancia euclídea al cuadrado), a los efectos de comparar las distribuciones de los individuos y evaluar si las tendencias observadas a través de ambos análisis son congruentes, lo que otorga 
una mayor robustez a las interpretaciones. Para llevar a cabo estos procedimientos se utilizaron los programas PAST versión 3.09 y SPSS versión 15.0.1.

\section{RESULTADOS}

En la Tabla 1 se indican los resultados obtenidos del análisis métrico de la dentición para cada uno de los individuos y en la Tabla 2, los valores correspondientes al análisis de componentes principales (ACP). De este conjunto de datos se desprende que los primeros tres componentes principales explican el $88,09 \%$ de la variabilidad total, por lo que resultan adecuados para graficar las distancias biológicas relativas entre cada individuo considerado.

Los resultados desde la métrica dental sugieren que no existirían diferencias importantes, entre los individuos analizados, que sean destacables en términos biológicos. Tanto los diagramas de dispersión (Figs. 3a, b) como el dendograma (Fig. 4) muestran una escasa dispersión de los valores entre los individuos y ninguna segregación agrupada de puntos, lo que sugiere cierta continuidad fenotípica durante el Holoceno tardío. Sin embargo, algunos individuos se separan, aunque levemente, del resto: PA4-47 (masculino), PA10A-21, LPE2 y PA10A-34 (femeninos). Asimismo, los individuos femeninos se distribuyen en forma relativamente más dispersa en relación a los masculinos (Figs. 3a y b). Se aplicó la prueba no paramétrica de Kolmogorov-Smirnov comparando la distribución de los individuos según el sexo para eva-

TABLA 2. Resultados del Análisis de Componentes Principales

\begin{tabular}{ccc}
\hline Componente & Eigenvalor & $\begin{array}{c}\text { Varianza } \\
\text { explicada (\%) }\end{array}$ \\
\hline 1 & 0,00857865 & 40,41 \\
2 & 0,00665256 & 28,59 \\
3 & 0,00566678 & 19,09 \\
4 & 0,00422015 & 5,96 \\
5 & 0,00174787 & 3,19 \\
6 & 0,00072763 & 1,59 \\
7 & 0,00060599 & 1,15 \\
8 & $6,3502 \mathrm{E}-06$ & 0,02 \\
\hline
\end{tabular}

luar la existencia de diferencias entre ambas submuestras. Los resultados indican que las mismas no son estadísticamente significativas $(\mathrm{Z}=0,34 ; \mathrm{p}=0,534)$.

\section{DISCUSIÓN Y CONCLUSIONES}

A partir de los análisis de la métrica dental se observó similitud en la dispersión de los valores. Si bien se reconocen problemas relacionados a la muestra, los resultados en principio permitirían inferir cierta continuidad fenotípica en los individuos durante el Holoceno tardío. En este sentido, podría tratarse de grupos biológicamente relacionados que inhumaron sus ancestros en el curso inferior del río Colorado durante todo ese período. Por el momento no es posible establecer si se trata una sola población a lo largo del tiempo o de varias poblaciones similares por procesos de mestizaje, o por ser grupos descendientes de un mismo ancestro común. Futuros análisis desde la biología molecular y la morfometría esqueletal podrán contribuir a discutir este problema.

$\mathrm{Si}$ se considera el factor sexo, no se observan discriminaciones claras, lo que indicaría que no habrían existido importantes diferencias biológicas entre individuos femeninos y masculinos, apoyando la tendencia arriba propuesta. De todos modos, la dispersión que presentan algunos individuos femeninos, permite sugerir una posible variabilidad biológica relativamente mayor que para los individuos masculinos (Fig. 3). Si bien no se observan casos que se separen significativamente del resto, se podría proponer a modo de hipótesis, que algunos de los individuos femeninos y un masculino podrían provenir de otras zonas geográficas o al menos pertenecer a grupos menos emparentados biológicamente con aquellos que habitaron la transición pampeano-patagónica oriental. Esta propuesta sólo podrá ser puesta a prueba cuando se disponga de un tamaño muestral mayor para su evaluación. Estudios genéticos (Postillone et al., 2016) y de isótopos estables en proceso permitirán aportar nueva información desde otras perspectivas para explorar si efectivamente existen diferencias en la procedencia de algunos individuos inhumados en el área de estudio.

Entre los individuos inhumados en el sitio Paso Alsina 1 no se observaron diferencias im- 


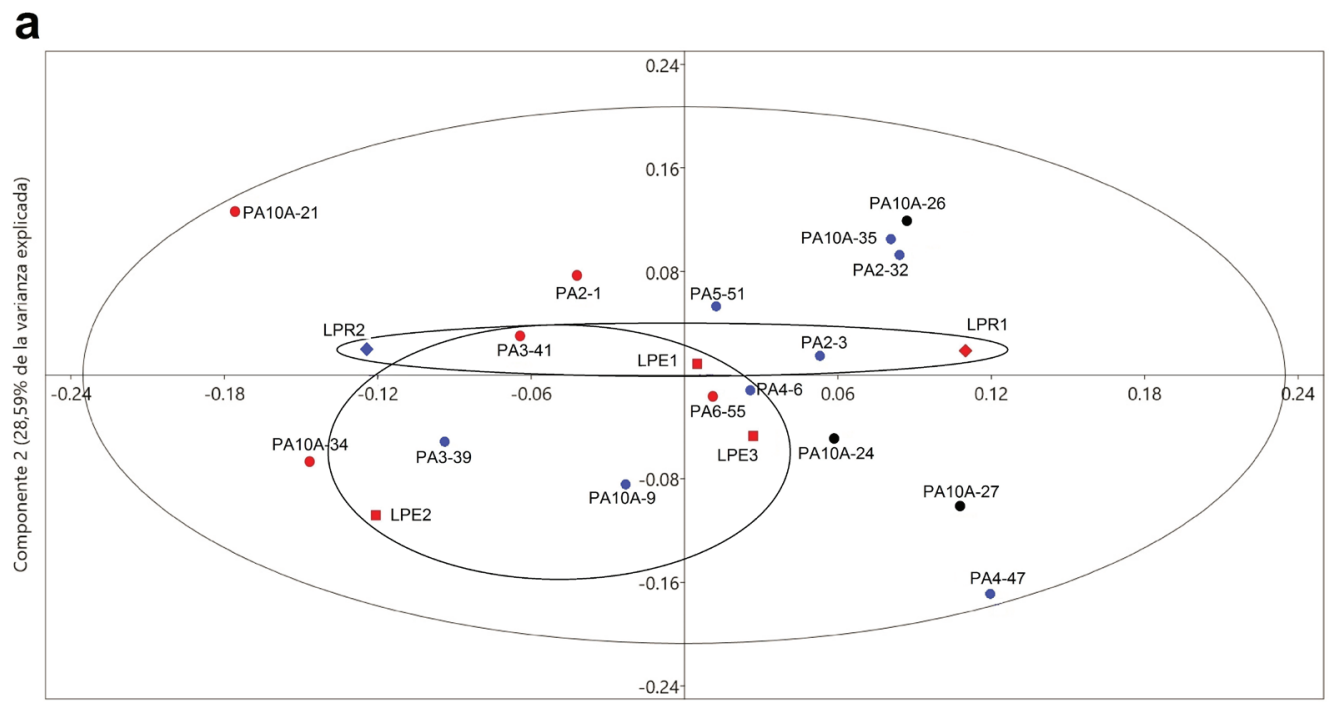

b

Componente $1(40,41 \%$ de la varianza explicada)

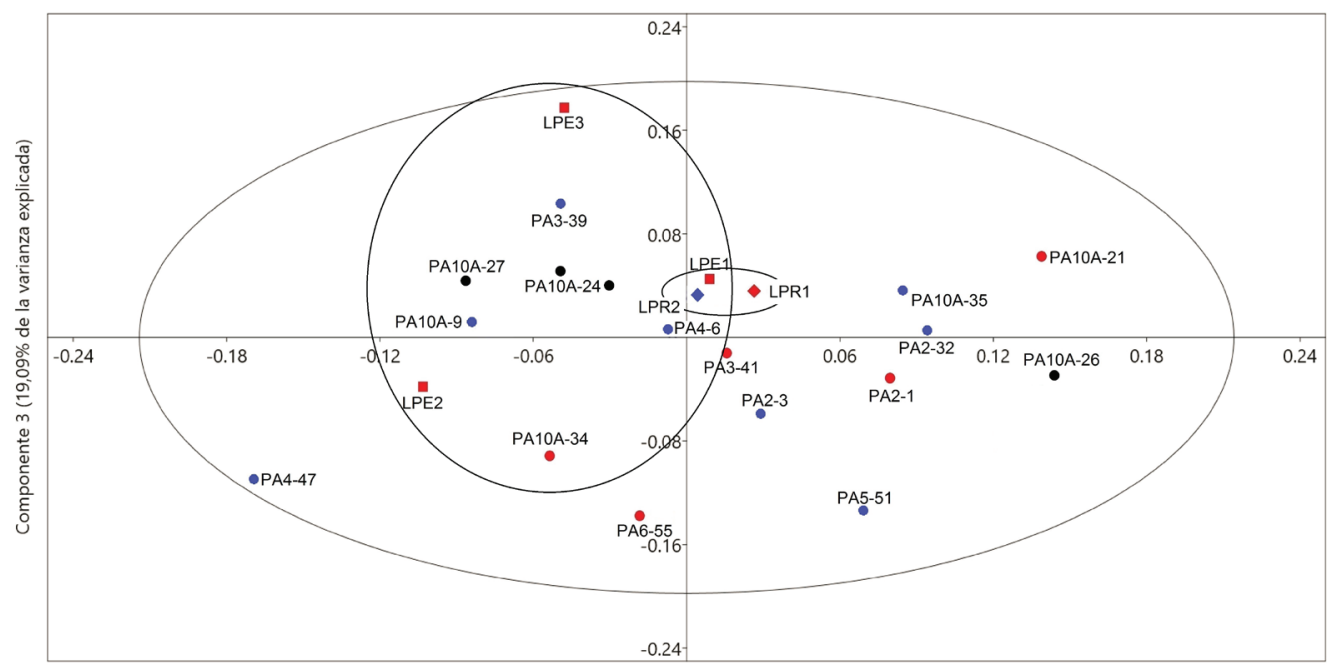

Componente $2(28,59 \%$ de la varianza explicada)

Fig. 3. Representación gráfica de los primeros tres componentes principales. Variabilidad total explicada: 88,09\%. Referencias: círculos: individuos del sitio Paso Alsina 1; cuadrados: individuos del sitio La Petrona; rombos: individuos del sitio La Primavera; azul: masculinos; rojo: femeninos; negro: sexo indeterminado. Elipses de confidencia al $95 \%$.

portantes, a excepción de PA4-47, que se aleja claramente del resto (Figs. 3a, b; Fig. 4). Por el momento, no es posible establecer una explicación adecuada para este caso debido a que no se registraron diferencias contextuales (e.g., modalidad de inhumación, cronología) ni individuales (e.g., patologías, deformaciones culturales). También es importante destacar que los individuos inhumados en un mismo entierro se distri- buyeron en diferentes sectores de los gráficos, lo que indica que no se identificaron agrupaciones de individuos por entierro (e.g., entierro 10A) y que aquellos que se asemejan entre sí forman parte de diferentes fardos funerarios (Figs. 3a, b; Fig. 4). Las investigaciones bioarqueológicas realizadas en esta compleja área formal de entierros secundarios, compuesta por una cantidad importante de individuos (Martínez et al., 2006, 


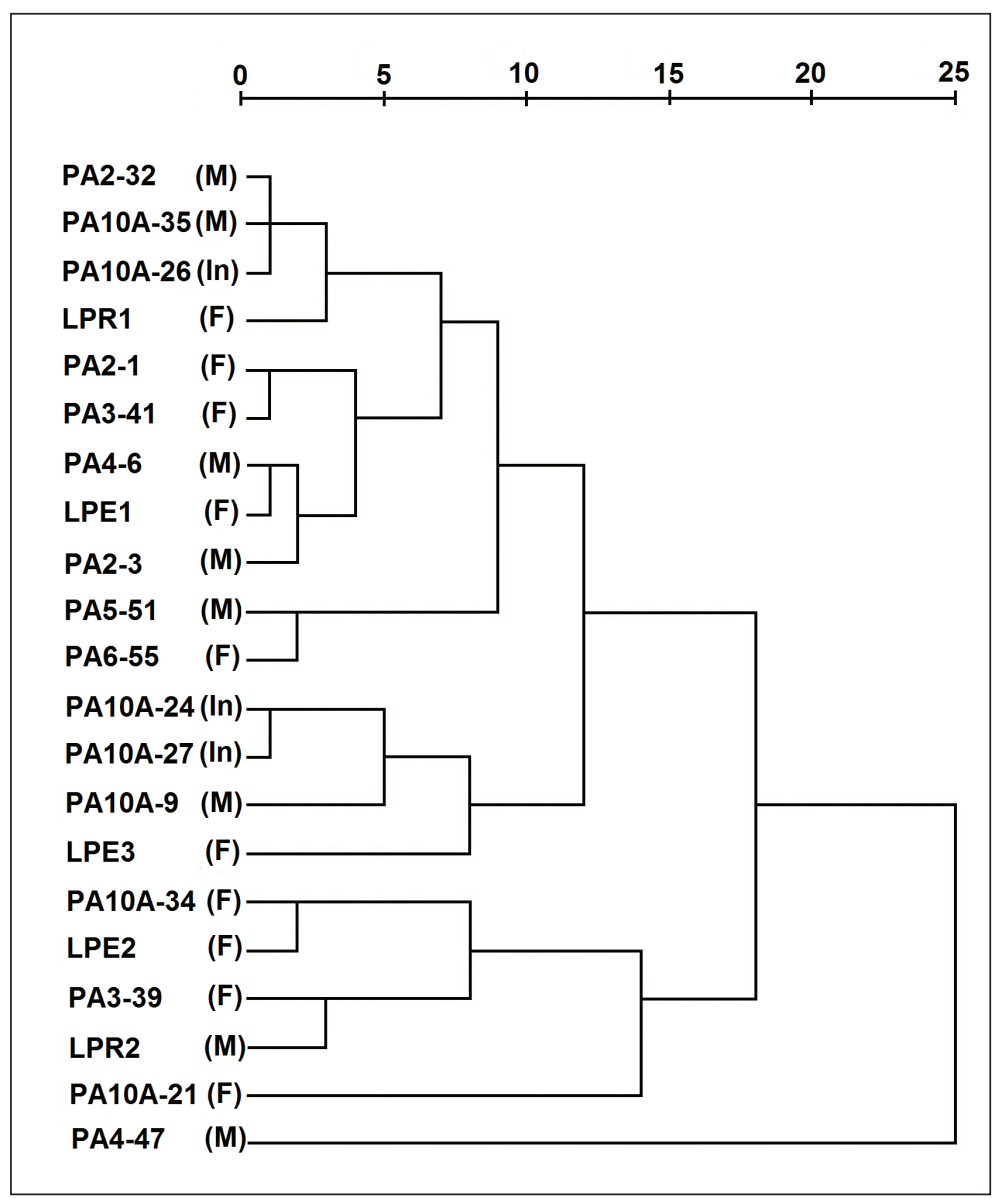

Fig. 4. Dendrograma jerárquico con la ubicación relativa de los individuos analizados (método de conglomeración de vinculación intergrupos, medida de distancia euclídea al cuadrado).

2012; González, 2013; Flensborg et al., 2015), permitieron proponer inicialmente que los individuos sepultados podrían pertenecer a diferentes grupos que confluyeron en tiempo y espacio para realizar el ritual mortuorio (Martínez et al., 2012). Sin embargo, el presente estudio en principio sugiere que no se trataría de poblaciones heterogéneas desde el punto de vista biológico. No obstante, este hecho no descarta la posibilidad de que, aun compartiendo cierta afinidad biológica, hubieran constituido grupos sociales diferentes provenientes de diversas áreas y que mantenían y fortalecían los lazos sociales a través de la re-inhumación de sus ancestros, además de participar activamente en el proceso de complementariedad y en las redes de interacción social (Kuijt, 1996; Chesson, 2009).

Desde una perspectiva comparativa regional, existen estudios métricos dentales realizados con una muestra procedente de sitios arqueológicos de Pampa Occidental. Luna (2008) analizó la dentición permanente de 26 individuos adultos y subadultos de ambos sexos en el sitio Chenque I, sitio La Lomita y sitio 3 de la Localidad Tapera Moreira, ubicados en el sur de la provincia de La Pampa y correspondientes a una cronología de 4500-400 años AP. Mediante el relevamiento de los diámetros mesiodistales y bucolinguales de la corona y del cuello se evaluó comparativamente el tamaño y las formas dentales en piezas uni y pluriradiculares. El autor observó diferencias estadísticas entre individuos que corresponderían a dos grupos fenotípicamente divergentes en el centro-sur de la provincia de La Pampa, durante ese lapso (Luna 2008). Estos resultados apoyan el modelo arqueológico propuesto pre- 
viamente por Berón (2004, 2007a, 2010), quien infirió la presencia de individuos pertenecientes al menos a dos poblaciones diferentes, a partir de un análisis multivariado que incluyó el sexo, la estatura, la modalidad de entierro y la presencia y tipo de deformación craneana intencional de 24 individuos adultos procedentes de los mismos sitios anteriormente mencionados. Asimismo, sugirió que estas dos poblaciones diferentes habrían habitado el sur pampeano entre $c a .4500$ y 400 años AP, interactuando solamente durante la última parte del período (Berón, 2007b). En base a toda la información disponible, Berón (2004) sostuvo que esta intensa dinámica poblacional puede ser explicada según el modelo de poblamiento regional propuesto por Barrientos (2001, 2002) para el Holoceno tardío desde la geografía evolutiva. Barrientos (2001) sugirió tres pulsos de poblamiento prehispánico en una amplia región que involucró la Pampa, el norte de Patagonia y las Sierras Centrales. El último pulso establecido en un rango de 4500-400 años AP habría impactado en la Pampa Occidental, principalmente durante el Holoceno tardío final (1000-400 años AP), período para el cual se propone la expansión de una población humana desde el área de Norpatagonia hacia las llanuras localizadas al norte, en la región Pampeana, y el reemplazo de poblaciones locales en el sudeste de la misma. Asimismo, Berón (2010) propuso la existencia de una complementariedad social y espacial entre ambas vertientes cordilleranas, utilizando las grandes cuencas hídricas de los ríos Colorado y Negro como conectores culturales y vías de desplazamiento en sentido oesteeste y viceversa. En consecuencia, diferentes procesos y dinámicas poblacionales que implicaron regiones muy disímiles operaron durante los últimos $c a$. 1000 años AP, generando una intensificación en las interacciones sociales a escala macroregional (Berón, 2007a, 2010).

Este escenario social y biológico propuesto para el sector meridional de la Pampa Occidental durante los últimos $c a$. 1000 años AP contrasta con el caso de estudio aquí presentado. En este sentido, en un área relativamente cercana, como es el curso inferior del río Colorado, el estudio de múltiples líneas de análisis derivadas del registro arqueológico y bioarqueológico permitieron proponer cambios organizacionales hacia la parte final del Holoceno tardío. Martínez (2008-
2009) sostiene una continuidad en la ocupación del área de estudio durante todo el Holoceno tardío, aunque con cambios organizacionales en distintas esferas como la subsistencia, el sistema de asentamiento y movilidad, la organización de la tecnología lítica y las prácticas funerarias durante el Holoceno tardío final. El autor propuso para ese período una situación de interacción y coexistencia social entre los grupos residentes y aquellos de regiones vecinas, en un área que habría formado parte de una "frontera blanda", de territorios permeables, habitados y negociados por grupos sociales provenientes de diferentes áreas y/o regiones. En un trabajo reciente (Martínez et al., 2017) se exploraron los cambios experimentados por las poblaciones cazadoras-recolectoras del noreste de Patagonia sobre la base del conocimiento obtenido del curso inferior del río Colorado como caso de estudio. Se consideraron factores demográficos, cambios en el patrón de asentamiento, subsistencia, tecnología (proveniencia de materias primas, circulación y uso de rocas), en la decoración de artefactos muebles, territorialidad, "packing" demográfico, circunscripción espacial, violencia interpersonal y complejidad en las prácticas mortuorias. El análisis de estas líneas llevó a plantear la existencia, para los últimos 1000 años AP, de sistemas sociales relativamente cerrados, que se expresan más intensamente hacia el $c a$. 500 años AP. Esto se habrían producido en un marco de relaciones sociales más competitivas ligadas a la construcción de procesos identitarios y de diferenciación social (véase también Mazzanti, 2006; Politis, 2008; Berón, 2012; Barberena, 2013). Estas transformaciones tuvieron su correlato en la existencia de procesos de regionalización con fuertes protocolos territoriales que influyeron en la movilidad, cobertura del paisaje, contactos y fluidez de bienes y personas (Martínez et al., 2017). Los resultados presentados aquí indican que no existirían diferencias importantes entre los individuos analizados en términos biológicos, sugiriendo continuidad entre el Holoceno tardío inicial y final. Dado el escenario social antes planteado para este último período, la homogeneidad intragrupal reconocida podría indicar que la intensidad de las relaciones sociales propuestas incluiría a individuos de la misma población biológica, aunque no se descarta que algunos proviniesen 
de áreas y regiones aledañas. Si bien es cierto que en un escenario de relaciones sociales relativamente cerradas los controles territoriales y el acceso a bienes y personas está mucho más controlado, la fluidez es esencial para la viabilidad de los grupos, lo que implica necesariamente intercambio de personas (Harris y Ross, 1987; Hitchcock y Bartram, 1998; Lourandos, 1997).

En sociedades de pequeña escala, las redes parentales son los principales canales de viabilización de las relaciones sociales que permiten sostener el pulso demográfico y que actúan y se estructuran según redes de derechos y obligaciones (Cane, 1990; Gamble, 1992). Sin embargo, deben considerarse también a los sistemas de alianzas, que exceden las relaciones estrictamente parentales y que son fundamentales para la renegociación y reciprocidad activa de las redes sociales. De hecho, los sistemas de alianzas contribuyen a la reproducción social y biológica (Gamble, 1982; Lourandos, 1997). En contextos de cazadores-recolectores con creciente grado de complejización, como los que se proponen durante el Holoceno tardío final en diferentes sectores de Pampa y Norpatagonia (Barrientos, 2001; Berón, 2007a; Martínez, 2008-2009), factores denso-dependientes tienen un rol importante en la estructuración de las relaciones entre grupos. Los ciclos de agregación, el intercambio de objetos y las uniones matrimoniales son necesarios para mantener los sistemas de alianzas y las redes ceremoniales (Lourandos, 1997; David y Lourandos, 1998; Hitchcock y Bartram, 1998; Gibbs y Veth, 2002). En consecuencia, tanto las relaciones de parentesco como las alianzas contribuyen a asegurar el intercambio de información, la generación de nuevos lazos parentales y funciona como un reaseguro ante diversas situaciones de estrés socio-ambiental (Gamble, 1982, 1992; Cane, 1990; Hitchcock y Bartram, 1998; Berón, 2004), eventos frecuentes en ambientes áridos-semiáridos como el área de estudio aquí presentada.

En suma, según los resultados aquí obtenidos, la interacción y complementariedad de diferentes grupos sociales que se propone para este lapso no habría implicado la interacción de poblaciones biológicamente diferentes, tal como es planteado para Pampa Occidental (Berón, 2007a, 2010; Luna, 2008). Este aspecto es en sí mismo destacable ya que podría estar indicando que durante el Holoceno tardío final (ca. 1000-250 años AP) se estarían desarrollando diferentes procesos y dinámicas poblacionales (e.g., regionalización, construcción de identidades, etc.) entre áreas y regiones vecinas (Béguelin et al., 2006; Mazzanti, 2006; Politis, 2008; Berón, 2012; Martínez et al., 2017).

De acuerdo con lo expuesto, este trabajo destaca la importancia de multiplicar los estudios de distancias biológicas desde una perspectiva biocultural en otras muestras bien documentadas de la región Pampeana y Patagónica. De este modo, se podrá profundizar en el conocimiento de la diversidad de los procesos sociales y biológicos que se desarrollaron durante el Holoceno tardío a nivel regional y de esa manera contribuir a la discusión acerca de la variabilidad de los patrones espacio-temporales identificados. Algunas de las ideas esbozadas en este trabajo son hipótesis de trabajo que deberán ser puestas a prueba mediante la incorporación de una mayor cantidad de individuos, principalmente correspondientes al Holoceno tardío inicial.

\section{AGRADECIMIENTOS}

Al INCUAPA-CONICET-UNCPBA por permitirnos llevar a cabo todos los análisis realizados en este trabajo. A Mariana Fabra por los comentarios de una versión previa de este artículo. A dos evaluadores anónimos, que con sus comentarios contribuyeron a mejorar el contenido de este trabajo.

\section{LITERATURA CITADA}

Alt K, Vach W. 1995. Odontologic kinship analysis in skeletal remains: concepts, methods, and results. For Sci Int 74:99-113. doi:10.1016/03790738(95)01740-A

Armentano G. 2016. Étude technologique des collections lithiques de nord-Patagonie orientale (Argentine) pendant l'Holocène récent. L'anthropologie 120:69-106. doi:10.1016/j.anthro.2016.02.001

Barberena R. 2013. Biogeografía, competencia y demarcación simbólica del espacio: modelo arqueológico para el norte de Neuquén. Intersecciones Antropol 14:367-381.

Barrientos G. 2001. Una aproximación bioarqueológica al estudio del poblamiento prehispánico tardío del Sudeste de la Región Pampeana. Intersecciones Antropol 2:3-18.

Barrientos G. 2002. The archaeological analysis of death-related behaviors from an evolutionary perspective: exploring the bioarchaeological 
record of early American hunter-gatherers. En: Martínez G, Lanata JL, editores. Perspectivas integradoras entre arqueología y evolución. Teoría, método y casos de aplicación. Serie Teórica $N^{0} 1$. Olavarría: INCUAPA, Universidad Nacional del Centro de la provincia de Buenos Aires. p 221253.

Barrientos G, Perez SI. 2004. La expansión y dispersión de poblaciones del norte de Patagonia durante el Holoceno tardío: evidencias arqueológicas y modelo explicativo. En: Civalero MT, Fernández P, Guráieb G, editores. Contra viento y marea. Arqueología de la Patagonia. Buenos Aires: INAPLSAA. p 1-17.

Barrientos G, Perez SI. 2005. Was there a population replacement during the Late mid-Holocene in the southeastern Pampas of Argentina? Archaeological evidence and paleoecological basis. Quatern Int 132:95-105. doi:10.1016/j.quaint.2004.07.018

Bayón C, Martínez G, Armentano G, Scabuzzo C. 2004. Arqueología del valle inferior del río Colorado: el sitio La Primavera. Intersecciones Antropol 5:39-53.

Béguelin M, Bernal V, Del Papa M, Novellino P, Barrientos G. 2006. El poblamiento humano tardío del sur de Mendoza y su relación con el norte de Patagonia: Una discusión bioarqueológica. Anales de Arqueología y Etnología 16:5-25.

Bernal V. 2007. Size and shape analysis of human molars: Comparing traditional and geometric morphometric techniques. Homo. J Comp Hum Biol 58:279-296. doi: 10.1016/j.jchb.2006.11.003

Bernal V. 2008. Procesos de diferenciación biológica entre poblaciones humanas del Holoceno tardío de Patagonia. Una aproximación desde la variación morfométrica dental. Tesis Doctoral Inédita. Facultad de Ciencias Naturales y Museo. Universidad Nacional de La Plata.

Bernal V, Perez SI, González P, Felizola Diniz-Filho J. 2010. Ecological and evolutionary factors in dental morphological diversification among modern human populations from southern South America. P Roy Soc Lond B Bio 277:1107-1112. doi:10.1098/rspb.2009.1823

Berón M. 2004. Dinámica poblacional y estrategias de subsistencia de poblaciones prehispánicas de la cuenca Atuel-Salado-Chadileuvú-Curacó, provincia de La Pampa. Tesis Doctoral Inédita. Facultad de Filosofía y Letras. Universidad de Buenos Aires.

Berón M. 2007a. Integración de evidencias para evaluar dinámica y circulación de poblaciones en las fronteras del Río Colorado. En: Morello F, Martinic M, Prieto A, Bahamonde G, editores. Arqueología de Fuego-Patagonia. Levantando piedras, desenterrando huesos... y develando arcanos. Punta Arenas: Ediciones CEQUA. p 173188.

Berón M. 2007b. Circulación de bienes como indicador de interacción entre las poblaciones de la Pampa Occidental y sus vecinos. En: Bayón C, González I, Flegenheimer N, Pupio A, Frére M, editores. Arqueología en las Pampas. Buenos Aires: Sociedad Argentina de Antropología. p 345 364.

Berón M. 2010. Circuitos regionales y conflictos intergrupales prehispánicos. Evidencias arqueológicas de violencia y guerra en la pampa occiden- tal Argentina. Actas del XVII Congreso Nacional de Arqueología Chilena I. Valdivia: Ediciones Kultrún, Sociedad Chilena de Arqueología, Universidad Austral de Chile. p 493-503.

Berón M. 2012. Patrones de violencia en sociedades preestatales: tipificación de eventos a partir de diferentes casos entre cazadores-recolectores de la Pampa occidental argentina. Una propuesta. En: López Mazz JL, Berón M, editores. Indicadores arqueológicos de violencia, guerra y conflicto en Sudamérica. Montevideo: Universidad de la República. p 81-115.

Black S, Ferguson E. 2011. Forensic anthropology 2000 to 2010. Boca Ratón: CRC Press.

Bollini G, Atencio JP, Colantonio S. 2012. Análisis odontométrico de cuatro series craneanas de aborígenes argentinos. Rev Arg Antropol Biol 14:6980. doi:10.17139/raab.2012.0014.1.25

Bollini J, Atencio JP, Luna LH. 2016. Caracterización de la dentición humana y aportes de la antropología dental para los estudios evolutivos, filogenéticos y adaptativos. En: Madrigal L, González-José $\mathrm{R}$, editores. Introducción a la antropología biológica. Buenos Aires: Asociación Latinoamericana de Antropología Biológica e Institute for the Study of Latin America and the Caribbean (ISLAC). p 618-648.

Buikstra J, Frankenberg S, Konigsberg L. 1990. Skeletal biology distance studies in American physical anthropology: recent trends. Am J Phys Anthropol 82:1-7. doi:10.1002/ajpa.1330820102

Butler P. 1937. Studies in the mammalian dentition and of teeth of Centetes ecaudatus and its allies. Proc Zool Soc Lond B107:103-132.

Butler P. 1939. Studies in the mammalian dentition and of differentiation of the postcanine dentition. Proc Zool Soc Lond B109:1-36.

Cane S. 1990. Desert demography: a case study of precontact aboriginal densities in the Western desert of Australia. En: Meehan B, White N, editores. Hunter-gatherer demography: past and present. Sydney: Oceania Monographs. p 149-159.

Chesson MS. 1999. Libraries of the dead: early Bronze age charnel houses and social identity at urban Bab edh-Dhra', Jordan. J Anthropol Archaeol 18:137-164. doi:10.1006/jaar.1998.0330

Christensen A. 1998. Odontometric microevolution in the valley of Oaxaca, Mexico. J Hum Evol 34:333-360. doi:10.1006/jhev.1997.0194

Cucina A, Lucci M, Vargiu R, Coppa A. 1999. Dental evidence of biological affinity and environmental conditions in prehistoric Trentino (Italy) samples from the Neolithic to the early Bronze age. Int J Osteoarchaeol 9:404-416. doi:10.1002/ (SICI)1099-1212

Dahlberg A. 1945. The changing dentition of man. J Am Dent Assoc 32:676-690. doi:10.14219/jada. archive.1945.0112

Darroch JN, Mosimann JE. 1985. Canonical and principal components of shape. Biometrika 72:241252. doi:10.1093/biomet/72.2.241

David B, Lourandos H. 1998. Rock art and socio-demography in northeastern Australian prehistory. World Archaeol 30(2):193-219.

Dempsey P, Townsend G. 2001. Genetic and environmental contributions to variation in human tooth size. Heredity 86:685-693. doi:10.1046/j.13652540.2001.00878.x 
Flensborg G. 2012. Análisis paleopatológico en el curso inferior del río Colorado (Pcia. de Buenos Aires). Exploración y evaluación del estado de salud de sociedades cazadoras-recolectoras en el Holoceno tardío. Tesis Doctoral Inédita. Facultad de Ciencias Sociales, UNICEN, Olavarría.

Flensborg G. 2015. Health and disease of hunter-gatherer groups from the eastern Pampa-Patagonia transition (Argentina) during the Late Holocene. Anthropol Sci 124(1):29-44. doi:10.1537/ase.151001

Flensborg G, Martínez G, Bayala P. 2015. Mortality profiles of hunter-gatherer societies: a case study from the Eastern Pampa-Patagonia transition (Argentina) during the final late Holocene. Int J Osteoarchaeol 25:816-826. doi:10.1002/oa.2348

Flensborg G, Martínez G, González M, Bayala P. 2011. Revisión de los restos óseos humanos del sitio La Petrona (transición pampeano-patagónica oriental, Argentina). Magallania 39:5-14. doi:doi. org/10.4067/S0718-22442011000100011

Gamble C. 1982. Interaction and alliance in Palaeolithic society. Man 17(1):92-107. doi: $10.2307 / 2802103$

Gamble C. 1992. Exchange, foraging and local hominid networks. En: Scarre C, Healey F, editores. Trade and exchange in prehistoric Europe. Oxford: Oxbow Books. p 35-44.

Garn S, Lewis A, Kerewsky R. 1967. Buccolingual size asymmetry and its developmental meaning. Angle Orthodont 37:186-193.

Gibbs M, Veth P. 2002. Ritual engines and territorial ascendancy. En: Ulm S, Westcott C, Reid J, Ross A, Lilley I, Prangnell J, Kirkwood L, editores. Borders, barriers and boundaries: proceedings of the 2001 Australian Archaeological Association Annual Conference. St. Lucia: University of Queensland. p 11-19.

González M. 2013. Procesos de formación y efectos tafonómicos en entierros humanos: el caso del sitio Paso Alsina 1 en Patagonia nororiental Argentina. Magallania 41(1):133-154. doi.org/10.4067/ S0718-22442013000100007

Hair J, Anderson R, Tatham R, Black C. 1999. Analisis multivariante. Madrid: Pearson Education.

Hanihara T, Ishida H. 2005. Metric dental variation of major human populations. Am J Phys Anthropol 128:287-298. doi:10.1002/ajpa.20080

Harris E, Lease L. 2005. Mesiodistal tooth crown dimensions of the primary dentition: a worldwide survey. Am J Phys Anthropol 128:593-607. doi:10.1002/ajpa.20162

Harris E, Nweeia, M. 1980. Tooth size of Ticuna indians, Colombia, with phenetic comparisons to other Amerindians. Am J Phys Anthropol 53:8191. doi:10.1002/ajpa.1330530112

Harris E, Potter R, Lin J. 2001. Secular trend in tooth size in urban Chinese assessed from two-generation family data. Am J Phys Anthropol 115:312318. doi:10.1002/ajpa.1087

Harris M, Ross E. 1987. La regulación demográfica en las sociedades preindustriales y en desarrollo. Madrid: Alianza Editorial.

Hillson S. 1996. Dental anthropology. Cambridge: Cambridge University Press.

Hillson S, FitzGerald C, Flinn H. 2005. Alternative dental measurements: proposals and relationships with other measurements. Am J Phys Anthropol 126:413-426. doi:10.1002/ajpa.10430
Hitchcock RK, Bartram LE. 1998. Social boundaries, technical systems, and the use of space and technology in the Kalahari. En: Stark MT, editor. The archaeology of social boundaries. Washington: Smithsonian Institution Press. p 12-49.

Jungers WL, Falsetti AB, Wall CE. 1995. Shape, relative size, and size-adjustments in morphometrics. Year Phys Anthropol 38:137-161. doi:10.1002/ ajpa. 1330380608

Kieser J. 2008. Human adult odontometrics. The study of variation in adult tooth size. Cambridge: Cambridge University Press.

Kuijt I. 1996. Negotiating equality through ritual: a consideration of late Natufian and prepottery Neolithic A period mortuary contexts. J Anthropol Archaeol 15:313-336. doi:10.1006/jaar.1996.0012

Latham K, Finnegan M. editores 2010. Age estimation of the human skeleton. Illinois: Charles C. Thomas.

Lourandos H. 1997. Continent of hunter-gatherers: new perspectives in Australian prehistory. Cambridge: Cambridge University Press.

Lukacs J. 1998. Human dental development, morphology, and pathology. A tribute to Albert A. Dahlberg. University of Oregon Anthropological Papers $\mathrm{N}^{\circ}$ 54. Oregon: University of Oregon Press.

Luna LH. 2006. Evaluation of uniradicular teeth for age-at-death estimations in a sample from a Pampean hunter-gatherer cemetery (Argentina). J Archaeol Sci 33:1706-1717. doi:10.1016/j. jas.2006.03.003

Luna LH. 2008. Estructura demográfica, estilo de vida y relaciones biológicas de cazadores-recolectores en un ambiente de desierto. Sitio Chenque I (Parque Nacional Lihué Calel, provincia de La Pampa). BAR International Series, vol. 1886. Oxford: Archaeopress.

Luna LH. 2010a. Alternative methodological procedures in sex determination of commingled and fragmentary human remains: an example from Argentine Pampean Region. En: Weiss K, editor. Trends in Physical Anthropology. Nueva York: Nova Science Publishers. p 1-48.

Luna LH. 2010b. Avances en la generación del perfil de mortalidad del sitio Chenque I: aportes de la antropología dental. En: Bárcena JR, Chiavazza $\mathrm{H}$, editores. Arqueología Argentina en el Bicentenario de la Revolución de Mayo I. XVII Congreso Nacional de Arqueología Argentina. Mendoza: Facultad de Filosofía y Letras, Universidad Nacional de Cuyo. p 243-246.

Luna LH. 2012. Validación de métodos para la generación de perfiles de mortalidad a través de la dentición. Su importancia para la caracterización paleodemográfica. Rev Arg Antropol Biol 14:33-51.

Luna LH. 2015. Interpretative potential of dental metrics for biodistance analyses in hunter-gatherers from Central Argentina. A theoretical-methodological approach. Homo. J Comp Hum Biol 66:432447. doi:10.1016/j.jchb.2015.03.001

Luna LH. 2016. Some achievements and challenges of dental anthropology. ARC J Dent Sci 1(1):5-9. doi:10.20431/2456-0030.0101003

Martínez G. 2008-2009. Arqueología del curso inferior del río Colorado: estado actual del conocimiento e implicaciones para la dinámica poblacional de cazadores-recolectores pampeano-patagónicos. Cazadores Recolectores del Cono Sur. Revista de Arqueología 3:71-92. 
Martínez G, Figuerero Torres MJ. 2000. Sitio arqueológico La Petrona (Pdo. de Villarino, Pcia. de Bs. As.): análisis de las modalidades de entierro en el área Sur pampeana. Relaciones XXV:227-247.

Martínez G, Bayala P, Flensborg G, López R. 2006. Análisis preliminar de los entierros humanos del sitio Paso Alsina 1 (Pdo. de Patagones, Pcia. de Buenos Aires). Intersecciones Antropol 7:95-108.

Martínez G, Flensborg G, Bayala P, López R. 2007. Análisis de la composición anatómica, sexo y edad de dos entierros secundarios del sitio Paso Alsina 1 (Pdo. de Patagones, Pcia. de Buenos Aires). En: Bayón C, Pupio A, González MI, Flegenheimer N, Frère M, editores. Arqueología en las Pampas. Buenos Aires: Sociedad Argentina de Antropología. p 45-58.

Martínez G, Flensborg G, Bayala P. 2012. Human corpse manipulation and the body as symbol: a case study from the Eastern Pampa-Patagonian transition (Argentina) during the Final Late Holocene. J Anthropol Archaeol 31:215-226. doi:10.1016/j.jaa.2011.12.002

Martínez G, Flensborg G, Bayala P. 2013. Chronology and human settlement in northeastern Patagonia (Argentina): patterns of site destruction, intensity of archaeological signal, and population dynamics. Quat Int 301:123-134. doi:10.1016/j. quaint.2013.03.027

Martínez G, Santos Valero F, Flensborg G, Carden N, Stoessel L, Alcaráz AP, Borges Vaz E. 2017. Was there a process of regionalisation in Northeastern Patagonia during the Late Holocene? J Isl Coast Archaeol 12(1):95-114. doi:10.1080/15564894.2 016.1163756

Matsumura M, Hudson M. 2005. Dental perspectives on the population history of Southeast Asia. Am J Phys Anthropol 127:182-209. doi:10.1002/ ajpa. 20067

Matsumura H, Oxenham M. 2014. Demographic transitions and migration in prehistoric east/southeast Asia through the lens of nonmetric dental traits. Am J Phys Anthropol 155:45-65. doi:10.1002/ ajpa. 22537

Mayhall J. 1992. Techniques for the study of dental morphology. En: Saunders S, Katzenberg M, editores. Skeletal biology of past peoples: research methods. Nueva York: Wiley-Liss. p 59-78.
Mayhall J. 2000. Dental morphology: techniques and strategies. En: Katzenberg M, Saunders S, editores. Biological anthropology of the human skeleton. Nueva York: Wiley-Liss. p 103-134.

Mazzanti D. 2006. La construcción de territorios sociales durante el Holoceno tardío. El caso de las sierras de Tandilia, Argentina. Relaciones 31:277-300.

Pietrusewsky M. 2000. Metric analysis of skeletal remains: methods and applications. En: Katzenberg M, Saunders S, editores. Biological anthropology of the human skeleton. Nueva York: Wiley-Liss. p 375-415.

Pinhasi R, Eshed V, Shaw P. 2008. Evolutionary changes in the masticatory complex following the transition to farming in the Southern Levant. Am J Phys Anthropol 135:136-148. doi:10.1002/ ajpa. 20715

Politis G. 2008. The pampas and campos of South America. En: Silverman H, Isbell W, editores. Handbook of South American Archaeology. Nueva York: Springer. p 235-260. doi:10.1007/978-0387-79407-5

Postillone B, Flensborg, Dejean C, Martínez G. 2016. Linajes maternos de los individuos del sitio arqueológico Paso Alsina 1 (Pdo. de Patagones, Buenos Aires). Actas XIX Congreso Nacional de Arqueología Argentina. San Miguel de Tucumán: Facultad de Ciencias Naturales e IML de la Universidad Nacional de Tucumán. p 1346-1348.

Rubin D. 1987. Multiple imputation for nonresponse in surveys. Nueva York: John Wiley.

Schafer JL. 2000. NORM: multiple imputation of incomplete multivariate data under a normal model, version 2.03. Software para Windows 95/98/NT. disponible en http://www.stat.psu.edu/jls/misoftwa.html

Scott G, Turner C. 1988. Dental anthropology. Ann Rev Anthropol 17:99-126. doi:10.1146/annurev. an.17.100188.000531

Scott G, Turner C. 2000. The anthropology of modern human teeth. Cambridge Studies in Biological and Evolutionary Anthropology, vol. 20. Cambridge: Cambridge University Press.

Stoessel L, Martínez G. 2014. El proceso de intensificación en la transición pampeano-patagónica oriental. Discusión y perspectivas comparativas con regiones aledañas. Comechingonia 18:65-94. 